\title{
Differences in Loading and Morphological Structure of the Take-off and Non-take-off Limb in Athletics Jumping Events
}

\author{
by \\ Petr Kutáč 1 , Jaroslav Uchytil ${ }^{1}$
}

The objective of the study was to assess differences between the take-off and non-take-off limbs of athletes in track-and-field jumping events based on a segmental analysis of body composition as well as kinetic analysis. The research included 19 participants (10 males, 9 females) with an average age of $18.1 \pm 2.8$ years. We measured body height, body mass, body composition (body fat, fat free mass, bone mineral content and bone density) and segmental distribution of these variables. To assess strength of the lower limbs, we performed reaction force analysis during takeoff and run symmetry. The difference in the representation of soft tissues between the take-off and non-take-off limbs was not significant; the differences were $0.06 \%, 0 \mathrm{~kg}$ in body fat and $0.01 \mathrm{~kg}$ in fat free mass. The differences in the values of bone matter were significant. The bone mineral content was $0.05 \mathrm{~kg}$ higher in the take-off limb $(p<0.001)$, and bone density was $0.07 \mathrm{~g} / \mathrm{cm} 2$ higher $(p<0.001)$; the practical significance of the difference was intermediate $(d=$ 0.5). Kinetic analysis showed that athletes exerted greater force on the pad with the take-off limb than the non-take-off limb when taking off while using arms in the first peak of the vertical force. The difference determined in this type of take-off was statistically significant $(p<0.05)$; the practical significance of the difference was medium $(d=0.7)$. The difference in the second peak of the vertical reaction force in the take-off arm was not statistically significant. The differences were reflected in the different bone matter compositions.

Key words: kinetics, body composition, segmental analysis, take-off limb.

\section{Introduction}

There are many sport disciplines where athletes' limbs perform a variety of activity during execution, such as the long jump and the high jump in track and field. In both of these events, performance technique is divided into four parts: the run-up, take-off, flight and landing (Bateman, 2004; Shepherd, 2013). Some authors state five phases in the long jump, adding a preparation phase between the run-up and takeoff (Nunn-Cearns, 2011). In either events, one of the lower limbs is the take-off limb or the limb that executes the take-off. A correctly executed take-off is a precondition for achieving peak performance. Therefore, long jumpers aim to get their centre of gravity into an optimal take-off angle (Bridgett and Linthorne, 2006; Shepherd,
2013), whereas high jumpers aim to get as high above the ground as possible (Panoutsakopoulos and Kollias, 2012). Additionally, the execution during performance as well as the load of the lower limbs will differ for each jump. In both jumping events, the lower limb the athlete uses for the take-off represents the dominant limb and is referred to as the take-off limb (Ae et al., 2008; Muraki et al., 2005). The description of proper technique in these events also confirms the significance of the take-off limb. Specifically, in the long jump, the take-off by the take-off limb follows a run-up along a straight runway towards the take-off board. When the take-off limb touches the take-off board, it is nearly fully extended, followed by a slight bending at the hip, knee and

1 - Human Motion Diagnostics Center, University of Ostrava, Ostrava, Czech Republic. 
ankle joints, finally followed by extension at these joints. This cycle is called accumulativerecuperation (Shepherd, 2013). The precondition for an effective take-off and thus overall performance during a long jump is sufficient and fast tension in the muscles (Zhao, 2000). The importance of the take-off limb is quite obvious in the high jump, although it follows a different technique than the long jump. The run-up starts on the opposite side of the take-off limb and progresses diagonally or slightly perpendicularly to the vertical plane of the crossbar at which point the athlete adjusts the last few steps from the runup line and commences a curve to the adjacent upright. The foot of the take-off limb does not land horizontally with the plane of the landing area, but it forms an angle of about $15-20^{\circ}$ with the vertical plane of the crossbar (Schiffer, 2009); the remaining steps in the execution of the takeoff limb are very similar to the long jump. The athlete lands on the extended take-off limb, followed with a slight bend and then stretching in all joints (McEwen, 2007). In the long jump, the take-off limb is flexed at the knee joint at an angle of about $175^{\circ}$ (Shepherd, 2013) and in the high jump it is $155^{\circ}$ (Ritzdorf, 2009). The description of the technique of both events clearly shows the different functions of the lower limbs, movement and load of the take-off limb. Sufficient force is required in order to get the centre of gravity into an optimal take-off angle in the long jump, and as high above the ground as possible in the high jump when the take-off is completed. Therefore, a question arises: is the reaction force manifested in the take-off greater in the take-off limb versus the non-take-off limb and are there any potential differences manifested in the body composition variables between the take-off limb and the nontake-off limb?

$$
\text { Some of the previous studies have }
$$
focused on the relationship between anthropometric variables and the height of the vertical jump (Aragon-Vargas and Gross 1997), the relationship between bone density and movement activity in various age groups (Arazi et al., 2016; Braun et al., 2015; Chastin et al., 2014), or the biomechanical analysis of jumping techniques (Lees et al., 1994; Letzelter, 2011; Van Gheluwe et al., 2003). However, the effect of long-term training in jumping events on the lower limbs of athletes has not been studied. Athletes in jumping events apply more loads on their take-off limb during training and so it can be expected that specific training would affect body composition and reaction force of the take-off and non-take-off limb.

The objective of this study was to assess the differences between the take-off and non-takeoff limb of athletes specializing in jumping events based on a segmental analysis of body composition and kinetic analysis of the take-off.

\section{Methods}

\section{Participants}

The study sample included 19 participants (10 males, 9 females) with an average age of $18.1 \pm$ 2.8 years. All of the participants were athletes involved in the long jump or high jump events. The inclusion criterion for participation in the research was the athlete's registration in a specialized preparation program in the evaluated event for at least a 2-year period, where they belonged to teams in their respective age categories.

Procedures

The segmental analysis of the body composition variables for the lower limbs was executed using the Dual-energy X-ray absorptiometry (DXA) method with a Hologic QDR Discovery densitometer (Hologic, Badford, USA). The measured variables were body fat (BF), fat free mass (LEAN), and bone mass. Bone mass is characterised by the values of bone mineral content (BMC) and bone mineral density (BMD). The measurement of lower limbs included the thigh, shin and foot. Figure 1 presents the definition of lower limb regions. The segmental analysis of bone mass of the lower limbs also included the measurement of the hip area, consisting of the hip joint and the proximal part of femoris axes (neck, trochanter). Figure 2 presents graphic illustration of the measured area.

Body mass (BM) was measured with the Tanita BC 418 MA tetrapolar bioimpedance scale (Tanita Corporation, Japan) and body height (BH) was evaluated using A-213 Anthropometer (Trystom, Czech Republic). Body height and mass were used as the initial variables for the densitometer measurements. All measurements were taken by the same experienced technician.

To assess strength of lower limbs, analysis of reaction force during the take-off was 
performed along with assessment of run symmetry. Two Kistler force plates (Kistler Instrumente AG, Wintherthur, Switzerland) connected to an analogue/digital (A/D) converter (5691A, Kistler Instrumente AG, Wintherthur, Switzerland) and a compatible computer were used to measure the impact force distribution. The frequency of force plates was $1200 \mathrm{~Hz}$. Prior to testing, the participants went through a total of 8 practice trials ( 2 for each leg and for each arm position). All the athletes executed the take-off without a run-up, only from one step for the takeoff and non-take-off limb, using arms, as well as with hands on the hips. The take-off method was selected after consultation with jumping events coaches. Each participant randomly performed 6 take-off trials using each leg and each arm position. The maximum values from the first and second vertical reaction force were evaluated. The first vertical force maximum was the value during the touchdown on the heel, i.e. absorption, and the second maximum was the value during the take-off from the point of the foot.

To assess the run, the participants ran on a 20-m long pavement with built-in force platforms. Speed was evaluated using wireless photocells and a trial was considered valid when each leg touched one platform and the speed was $3.2 \mathrm{~m} / \mathrm{s} \pm$ $5 \%$, so that the force variables would not be influenced by speed. Each athlete performed 6 valid trials. The maximum of forces in the vertical and anteroposterior direction were evaluated. In the anteroposterior direction, the first maximum was the value of the highest force in the braking phase of the step, and the second maximum was the value of the highest force in the acceleration phase of the step cycle.

The force values were normalised to body mass of the participant and they were expressed in body weight percentage ( $\% \mathrm{BW})$.

In all cases, both the take-off and non-takeoff limbs were measured so that the measured variables could be compared.

\section{Statistical Analysis}

To assess statistical significance of the differences between the take-off and non-take-off limbs, a paired sample t-test was used. The level of statistical significance was selected at $p=0.05$ for all applied tests. Practical significance was also determined for values that were statistically significant by calculating effect size (ES) using
Cohen's $d$ guidelines. The $d$ value at the level of 0.2 indicated a minor change, 0.5 an intermediate change and 0.8 a major change (Cohen, 1988). The accuracy of the densitometer measurement was assessed in variables with both statistic and practical significance. To determine the accuracy of measurement, we used the typical error of measurement (TE) recommended by Hopkins (2000). The author indicated this method of expressing the accuracy of measurement for biomedical branches. According to the recommendations, three repeated measurements immediately following one another were taken in 20 people without any medical issues. TE value was calculated on the basis of the square root of the scalar product of square roots of typical errors $\left(T E^{2}\right)$ between the pairs of trials and degrees of freedom $(\mathrm{Df}=19)$ divided by the total sum of the degrees of freedom.

Resulting TE: $\frac{\sqrt{\sum_{i} T E_{i}^{2} \cdot D f_{i}}}{\sum_{i} D f_{i}}$

Considering this was a repeated measurement design, we calculated intraclass correlation coefficients (ICC). The results of the accuracy of measurement and correlations are presented in Table 3. The table also contains descriptive statistics for the vertical force and variables of body composition (i.e., mean and standard deviation). The force was normalized to body mass of the individual participants and expressed in $\%$ of body weight (\% BW).

The results were statistically processed using IBM SPSS Statistics (Version 21; IBM, Armonk, NY, USA).

All participants signed an informed consent form for their participation in this research. The research was approved by the Ethics Committee of the University of Ostrava and complied with the Declaration of Helsinki for medical research.

\section{Results}

The somatic characteristics and mean values of body composition analysis are presented in Table 1.

The values of body composition variables of the take-off and non-take-off limbs are presented in Table 2. The values for the vertical forces during the take-off from the take-off and 
non-take-off limbs are presented in Table 4. The tables present the mean values measured for the lower limbs of both genders $(n=19)$. We did not consider it important to distinguish between genders while assessing the differences between the take-off and non-take-off limbs, as gender is unlikely to impact final results.

When evaluating the segmental analysis of the limb composition, we found statistically significant differences between the take-off and non-take-off limbs only for the bone mass fraction $(p<0.001)$. The BMC and BMD values were higher in the take-off limb. The practical significance of those variables was in the moderate range $(d=$ 0.5 ). The differences found (50 g BMC and 0.07 $\mathrm{g} / \mathrm{cm}^{2} \mathrm{BMD}$ ) were also higher than both the TE and the upper confidence limit values (Table 3). The determined ICC values confirmed high accuracy of measurement as they explained 96 to $98 \%$ of the variance.

The kinetic analysis showed that the athletes exerted a greater force on the pad during the take-off with arms in the first peak of the vertical force for the take-off limb than for the non-take-off limb. This type of the take-off showed a statistically significant difference $(p<$ $0.05)$; practical significance was intermediate $(d=$ $0.7)$. The difference in the second peak of the vertical reaction force in the take-off using arms was not statistically significant. No statistically significant differences were detected during takeoffs with fixed arms in the first or second peak of the vertical reaction force. The mean values of the vertical reaction force were greater for the take-off limb during the entire course of the take-off limb compared to the non-take-off limb for both upper limbs (Figure 1) and with fixed limbs (Figure 2). The difference in reaction forces in the vertical and anteroposterior direction between the take-off and non-take-off limb while running was not statistically significant (Table 4).

Table 1

\begin{tabular}{|c|c|c|}
\hline & Male $(n=10)$ & Female $(\mathrm{n}=9)$ \\
\hline Variables & $\mathrm{M} \pm \mathrm{SD}$ & $\mathrm{M} \pm \mathrm{SD}$ \\
\hline $\mathrm{BM}(\mathrm{kg})$ & $69.20 \pm 4.85$ & $61.67 \pm 6.48$ \\
\hline $\mathrm{BH}(\mathrm{cm})$ & $181.00 \pm 4.94$ & $175.13 \pm 5.97$ \\
\hline $\mathrm{BF}(\%)$ & $14.42 \pm 2.33$ & $25.09 \pm 2.16$ \\
\hline $\mathrm{BF}(\mathrm{kg})$ & $9.91 \pm 1.80$ & $15.38 \pm 2.27$ \\
\hline LEAN (kg) & $55.69 \pm 3.74$ & $43.35 \pm 4.50$ \\
\hline $\mathrm{BMC}(\mathrm{g})$ & $3036.30 \pm 328.03$ & $2477.56 \pm 190.59$ \\
\hline $\operatorname{BMD}\left(\mathrm{g} / \mathrm{cm}^{2}\right)$ & $1.31 \pm 0.12$ & $1.19 \pm 0.07$ \\
\hline
\end{tabular}


Table 2

Segmental analysis of the body composition of lower limbs

\begin{tabular}{|c|c|c|c|c|}
\hline \multirow[t]{2}{*}{ Variables } & $\begin{array}{l}\text { TLimb } \\
(\mathrm{n}=19)\end{array}$ & $\begin{array}{l}\text { NTlimb } \\
(\mathrm{n}=19)\end{array}$ & \multirow{2}{*}{ Diff } & \multirow[t]{2}{*}{$d$} \\
\hline & $\mathrm{M} \pm \mathrm{SD}$ & $\mathrm{M} \pm \mathrm{SD}$ & & \\
\hline BF (\%) & $21.47 \pm 8.51$ & $21.53 \pm 8.51$ & $0.06^{\mathrm{NS}}$ & - \\
\hline BF (kg) & $2.74 \pm 1.10$ & $2.74 \pm 1.07$ & $0.00^{\mathrm{NS}}$ & - \\
\hline LEAN (kg) & $9.50 \pm 1.64$ & $9.51 \pm 1.65$ & $0.01^{\mathrm{NS}}$ & - \\
\hline $\mathrm{BMC}(\mathrm{g})$ & $620.79 \pm 111.18$ & $570.74 \pm 104.69$ & $50.05^{* * *}$ & 0.5 \\
\hline $\operatorname{BMD}\left(\mathrm{g} / \mathrm{cm}^{2}\right)$ & $1.48 \pm 0.16$ & $1.41 \pm 0.15$ & $0.07^{* * *}$ & 0.5 \\
\hline
\end{tabular}

Hip area

\begin{tabular}{lccll}
\hline BMC $(\mathrm{g})$ & $49.85 \pm 11.41$ & $48.08 \pm 10.51$ & $1.77^{\mathrm{NS}}$ & - \\
$\mathrm{BMD}\left(\mathrm{g} / \mathrm{cm}^{2}\right)$ & $1.20 \pm 0.14$ & $1.20 \pm 0.13$ & $0.00^{\mathrm{NS}}$ & - \\
\hline
\end{tabular}

BF - body fat, LEAN - fat free mass, BMC - bone mineral content, BMD - bone density, TLimb - take-off limb, NTLimb - non take-off limb, $n$-frequency, Diff-difference, $d$-effect size, $M-$ mean, $S D$ - standard deviation, NS - not significant, and ${ }^{* * *} p<0.001$

Table 3

Accuracy measurements by DXA

\begin{tabular}{lcccccc}
\hline \multirow{3}{*}{ Variables } & \multicolumn{2}{c}{ Trial 2-1 } & \multicolumn{2}{c}{ Trial 3-2 } & Mean \\
\cline { 2 - 7 } & TE & ICC & TE & ICC & TE & ICC \\
& $(95 \%$ CI $)$ & & $(95 \%$ CI $)$ & & $\mathbf{( 9 5 \% ~ C I ) ~}$ & \\
\hline BMC LL (g) & 10.69 & 0.99 & 9.86 & 0.99 & $\mathbf{1 0 . 2 4}$ & $\mathbf{0 . 9 9}$ \\
& $(8.49 / 14.65)$ & & $(7.83 / 13.51)$ & & $\mathbf{( 8 . 5 4 / 1 3 . 2 4 )}$ &
\end{tabular}

\begin{tabular}{lcccccc}
\hline BMC RL (g) & 17.01 & 0.98 & 16.64 & 0.98 & $\mathbf{1 6 . 7 5}$ & $\mathbf{0 . 9 8}$ \\
& $(13.50 / 23.31)$ & & $(13.21 / 22.81)$ & & $\mathbf{( 1 3 . 9 8 / 2 1 . 6 7 )}$ &
\end{tabular}

\begin{tabular}{lcccccc}
\hline BMD LL (g/cm²) & 0.01 & 0.99 & 0.02 & 0.99 & $\mathbf{0 . 0 2}$ & $\mathbf{0 . 9 9}$ \\
& $(0.01 / 0.02)$ & & $(0.02 / 0.03)$ & & $\mathbf{( 0 . 0 1 / 0 . 0 2 )}$ & \\
& & & & & & \\
\hline BMD RL (g/cm $)$ & 0.02 & 0.99 & 0.03 & 0.98 & $\mathbf{0 . 0 3}$ & $\mathbf{0 . 9 8}$ \\
& $(0.02 / 0.03)$ & & $(0.02 / 0.04)$ & & $\mathbf{( 0 . 0 2 / 0 . 0 3 )}$ &
\end{tabular}

$B M C$ - bone mineral content, BMD - bone density, LL Leg - left leg, RL - right leg, TE - typical error, 95\% CI - confidence interval, ICC - intraclass correlation 
Table 4

Comparison of vertical forces for the take-off and idle limbs

\begin{tabular}{|c|c|c|c|c|}
\hline \multirow[t]{2}{*}{ Force $(\% \mathrm{BW})$} & $\begin{array}{l}\text { TLimb } \\
(\mathrm{n}=19)\end{array}$ & $\begin{array}{c}\text { NTLimb } \\
(\mathrm{n}=19)\end{array}$ & \multirow[t]{2}{*}{ Diff } & \multirow[t]{2}{*}{$d$} \\
\hline & $\mathrm{M} \pm \mathrm{SD}$ & $\mathrm{M} \pm \mathrm{SD}$ & & \\
\hline Arm take-off 1st peak & $1.76 \pm 0.61$ & $1.39 \pm 0.43$ & $0.37^{*}$ & 0.7 \\
\hline Arm take-off 2 nd peak & $3.01 \pm 0.42$ & $2.89 \pm 0.69$ & 0.12 & \\
\hline Fix arm take-off $1^{\text {st }}$ peak & $1.98 \pm 0.59$ & $1.75 \pm 0.56$ & 0.23 & \\
\hline $\begin{array}{l}\text { Fix arm take-off } 2^{\text {nd }} \\
\text { peak }\end{array}$ & $2.51 \pm 0.38$ & $2.49 \pm 0.47$ & 0.02 & \\
\hline Vertical peak running & $2.21 \pm 0.32$ & $2.19 \pm 0.31$ & 0.15 & \\
\hline AP $1^{\text {st }}$ peak running & $0.27 \pm 0.04$ & $0.27 \pm 0.05$ & 0.28 & \\
\hline $\mathrm{AP} 2^{\text {nd }}$ peak running & $-0.25 \pm 0.16$ & $-0.28 \pm 0.06$ & 0.25 & \\
\hline
\end{tabular}

TLimb-take-off limb, NTLimb-non take-off limb, $n$-frequency, Diff-difference, $d$-effect size, $M-$ mean, $S D-$ standard deviation, NS - not significant, and ${ }^{*} p<0.05$

Figure 1

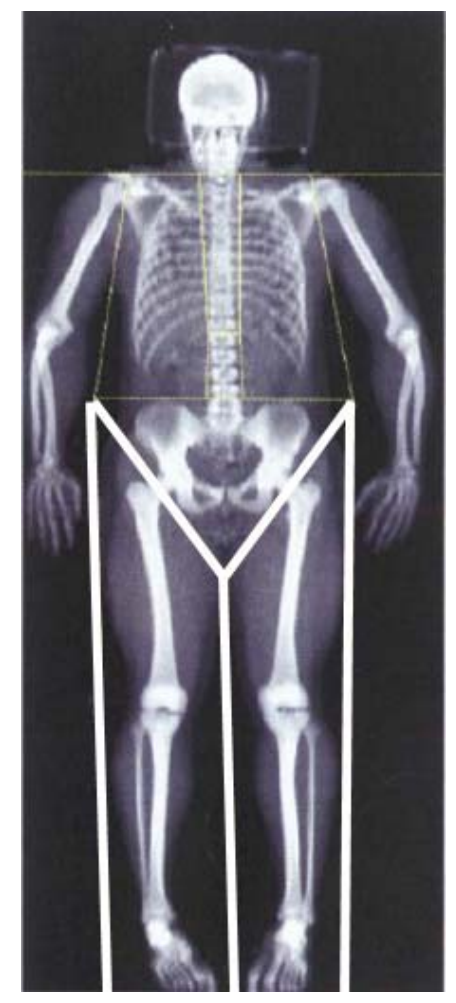

Area of the lower limb measurements 


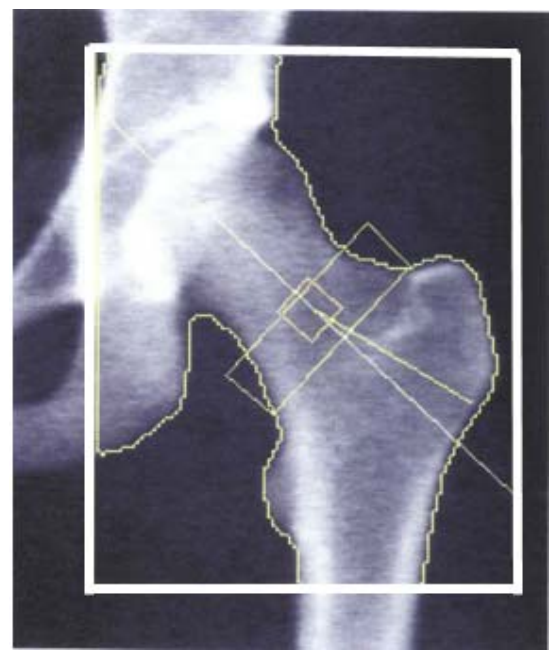

Figure 2

Hip area of the bone mass measurement

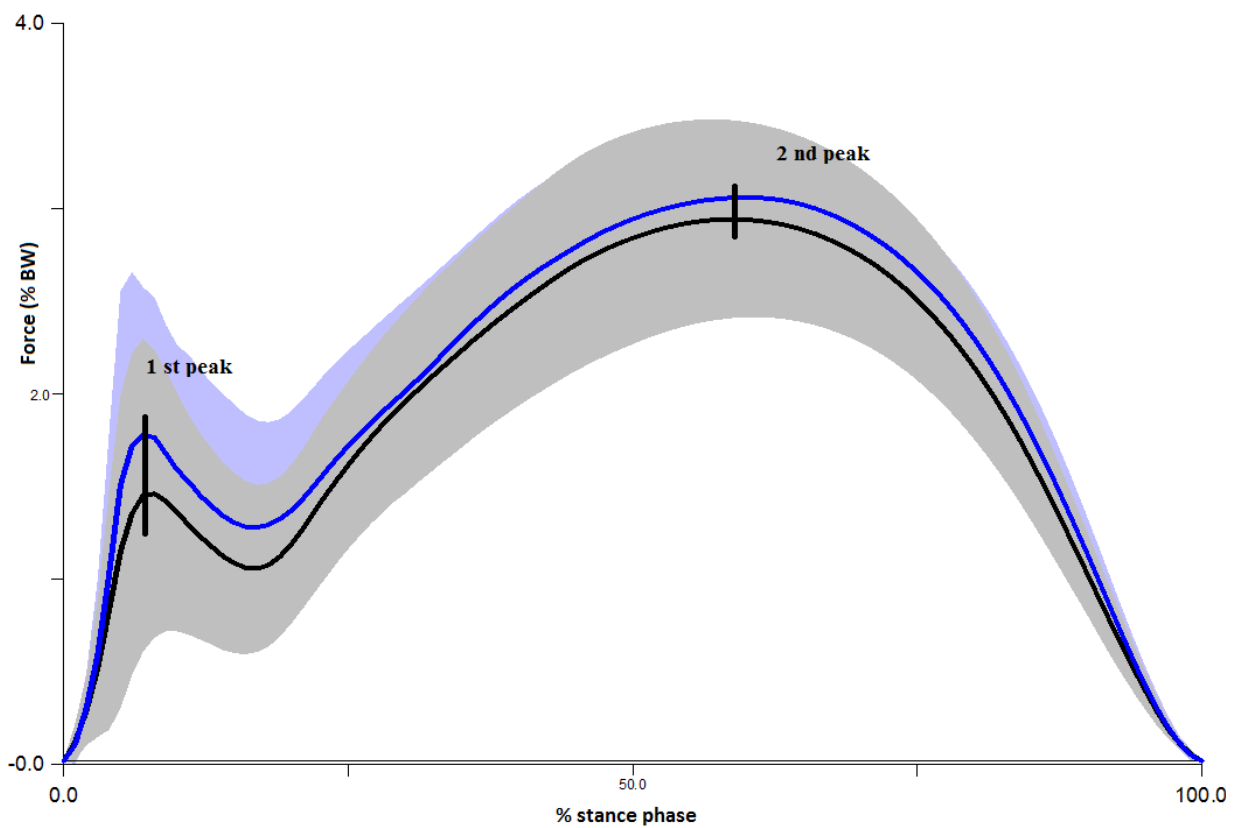

Figure 3

Course of the reaction force during take-off with the use of arms 


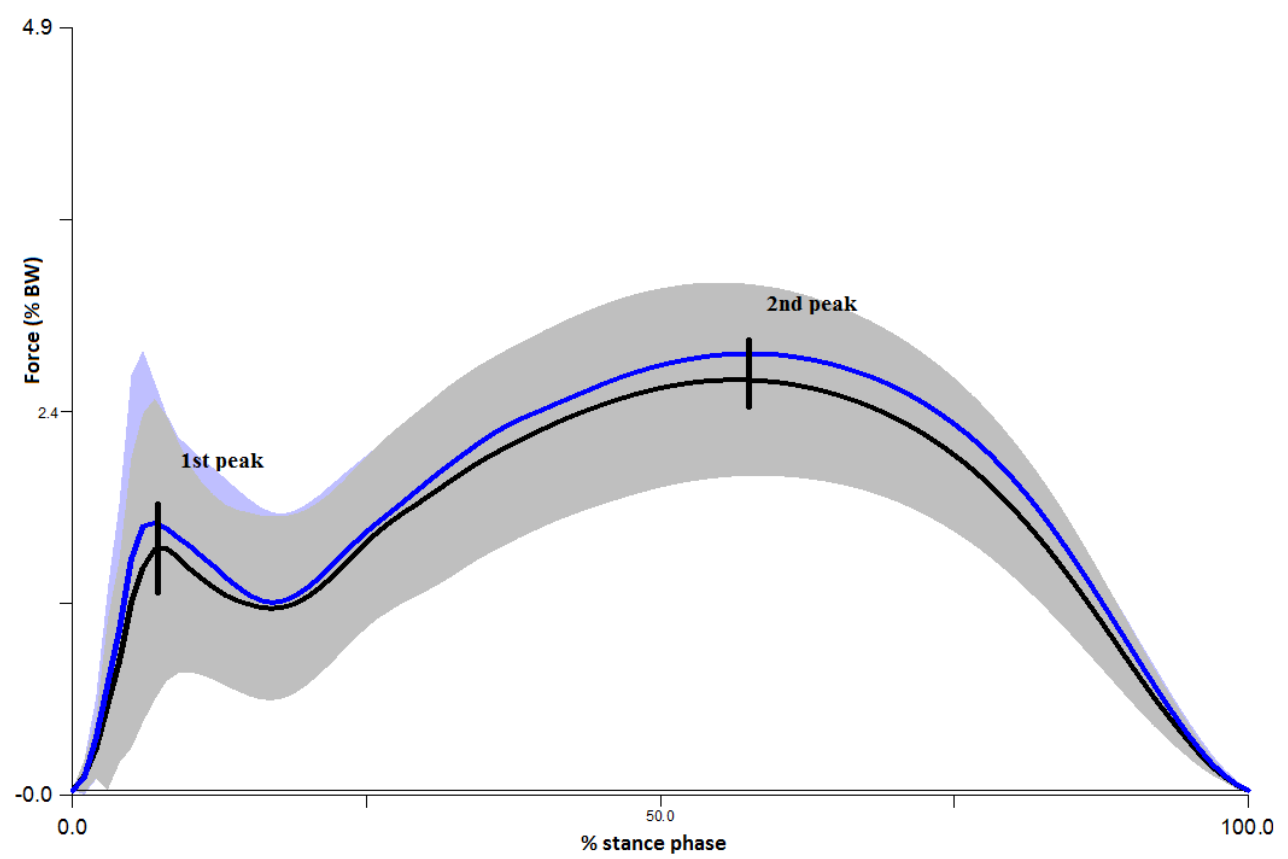

Figure 4

Course of the reaction force during take-off without the use of arms

\section{Discussion}

Based on the segmental analysis of soft tissue (BF, LEAN) in lower limbs, the distribution of BF and LEAN between the take-off and nontake-off limbs appeared balanced. The differences were negligible despite the fact that one of the limbs was used for the take-off and should thus be exposed to a higher load. This lack of a difference may be caused by applying a similar reaction force on the take-off and non-take-off limbs during the take-offs. The differences in limb composition were even lower than in other sports, in which the resulting performance is not affected by the take-off limb. For example, in ice-hockey players, the differences in the percentage of $\mathrm{BF}$ between the right and left limbs ranged from 0.26 to $0.42 \%$ BF (Kutáč, 2012; Kutáč and Sigmund, 2015), whereas in soccer players, they ranged from 0.3 to $0.88 \%$ BF (Kutáč, 2011). The presented results imply that preparation of athletes is executed on the basis of scientific principles, with emphasis on balanced and symmetrical development. This principle should apply to all sport disciplines and fields. The segmental analysis of bone mass (BMC, BMD) in the lower limbs showed a higher ratio of bone mass and higher density in the take-off limb. The differences are most likely caused by impacts incurred when the take-off limb touches the takeoff board in the long jump, or when the stretched take-off limb touches the ground during the last step in the high jump. The accuracy of this substantiation has been confirmed by Shephard (2013) and Ritzdorf (2009) who state that the supporting and locomotor apparatus of the takeoff limb is overloaded during the long jump and high jump. The higher load of the take-off limb is also confirmed by the higher value of the first maximum of the vertical reaction force of this limb during the take-off when using arms. The value of the first maximum occurs in the initial 
phase of energy absorption prior to the take-off. The effect of impacts on the increase in the bone mass ratio for the take-off lower limbs of athletes has been also confirmed by Heinonen et al. (2001) who compared the values of bone mass in lower limbs in their study using the DXA method and peripheral quantitative computed tomographic analysis in triple jumpers and in a control group. The bone mass values in triple jumpers was significantly higher compared to the control group. The effect of repeated impacts on the increase of bone mass, which is a sign of bone remodelling, has been also confirmed in a study dealing with the effect of jump training on the increase of BMD in young women (Kato et al., 2006). The experimental group of young women in the study went through high impact training (10 maximum vertical jumps/day, 3 times/week) for a period of 6 months. After the end of impact training, the authors used the DXA method to compare changes in the values of bone density. The experimental group showed increased BMD in the areas of the lumbar spine and femoral neck. In our study, analysis of changes in the representation of bone mass (BMC, BMD) in the lower limbs of the jumpers that we monitored showed that unlike the results of the study by Kato et al. (2006), there was no change caused by the higher effect of impacts on the take-off limb in the femoral neck area. The differences between BMC and BMD in the hip area were not significant (Table 2). This fact is explained by the result of the biomechanical analysis in the course of the take-off, which showed that the take-off limb bore more loading during the first phase of the take-off, i.e., absorption phase, when the structures in the area of the crus were engaged. That may be the reason that no differences in BMC and BMD were found in the hip area. The importance of the high impact exercise program was also confirmed by Illianca et al. (2010) who recommended it as prevention against changes in the structure of bones and the occurrence of osteoporosis. The effect of impact during repeated take-offs and landings on the lower limb BMC values was demonstrated by Schinkel-Ivy et al. (2014). The authors in the study compared BMC values of lower limbs in athletes of various disciplines with different frequency of take-offs and landings using standardised anthropometric measurements and prediction equations. Lower limbs were defined as the area between the knee joint centre, lateral and medial malleoli, and foot segments. The results showed that the highest BMC values were found in volleyball athletes, followed by basketball athletes, soccer athletes, and cross-country athletes having the lowest values. This order corresponds with the frequency of take-offs and landings in those sports.

\section{Conclusion}

The results of the body composition segmental analysis of lower limb segments show that loading of both lower limbs during trackand-field jumps is balanced. There is a difference only in the take-off phase, which is confirmed by the differences between the take-off and non-takeoff limbs in terms of the reaction force. Those differences exist during the entire course of the take-off; however, there is only a statistically significant difference for the first maximum of the reaction force in the accumulative phase of the take-off. The load generated on the take-off limb is not sufficient to affect different representations of the individual soft tissues in the take-off and non-take-off limbs. The only difference was found in the values of bone mass content and bone density in the foot and shin regions, which correspond to the differences in the reaction forces. In the training process, coaches should also focus on the execution of specific take-off exercises when using the idle lower limb.

\section{Acknowledgements}

The present research material is original and has not been submitted elsewhere for publication. The research was funded by the Research Projects: SGS 6167/PdF 2015-2016 The Effect of Asymmetric Limb Loading on Limb Strength, Joint Kinetics and Kinematics and Segmental Body Fat Distribution; and Program 4 HAIE - Healthy Aging in Industrial Environment (CZ.02.1.01/0.0/0.0/16_019/0000798). 


\section{References}

Ae M, Nagahara R, Ohshima Y, Koyama H, Takamoto M, Shibayama K. Biomechanical analysis of top three male high jumpers at the 2007 World Championships in Athletics. New Studies in Athletics, 2008; 23: 4552

Arazi H, Eghbali E, Saeedi T, Moghada R. The Relationship of Physical Activity and Anthropometric and Physiological Characteristics to Bone Mineral Density in Postmenopausal Women. J Clin Densitom, 2016; 19: 382-388

Aragon-Vargas LF, Gross MM. Kinesiological factors in vertical jump performance: differences among individuals. J Appl Biomech, 1997; 13: 24-44

Bateman T. High jump styles within the Flop technique. Coach. 2004; 22: 943-46

Braun SI, Kim Y, Jetton AE, Kang M, Morgan DW. Prediction of bone mineral density and content from measures of physical activity and sedentary behavior in younger and older females. Preventive Medicine Reports, 2015; 2: 300-305

Bridgett L, Linthorne N. Changes in long jump take-off technique with increasingrun-up speed. J Sport Sci, 2006; 24: 889-897

Chastin SF, Mandrichenko O, Helbostadt JL, Skelton DA. Associations between objectively-measured sedentary behaviour and physical activity with bone mineral density in adults and older adults, the NHANES study. Bone, 2014; 64: 254-262

Cohen J. Statistical power analysis for the behavioral sciences. New Jersey: Lawrence Erlbaum Associates, 273288; 1988

Heinonen A, Sievänen H, Kyröläinen H, Perttunen J, Kannus P. Mineral mass, size, and estimated mechanical strength of triple jumpers' lower limb. Bone, 2001; 29: 279-285

Hopkins WG. Measures of Reliability in Sports Medicine and Science. Sports Med 2000; 30: 1-15

Ilianca I, Avramescu T, Shaao M, Rosulescu E, Zavaleanu M. The Role Of High - Impact Exercise in Improve Bone Mineral Density in Postmenopausal Women With Osteopenia or Osteoporosis. Journal of Physical Educational \& Sport, 2010; 27: 110-116

Kato T, Tereshima T, Yamashita T, Hatanaka Y, Honda A, Umemura Y. Effect of low-repetition jump training on bone mineral density in young women. J App Physiol, 2006, 100: 839-843

Kutác P. The effect of the measuring mode on the results of body composition using bioelectrical impedance method. Česká Antropologie, 2011; 61: 28-32

Kutáč P. Development of Somatic Parameters of Ice Hockey Players. Česká antropologie, 2012; 62: 9-14

Kutáč P, Sigmund M. A Comparison of Somatic Variables of Elite Ice Hockey Players from the Czech ELH and Russian KHL. J Hum Kinet, 2015; 45: 189-197

Lees A, Graham-Smith P, Fowler N. A biomechanical analysis of the last stride, touch-down and take-off characteristics of the men's long jump. J Appl Biomech, 1994; 10: 61-78

Letzelter S. The importance of horizontal and vertical take-off velocity for elite female long jumpers. New Studies in Athletics, 2011; 26: 73-84

McEwen F. High Jump: Teaching the Fosbury Flop. Modern Athlete \& Coach, 2007; 45: 10-14

Muraki Y, Yokozawa T, Ae M, Koyama H. Athletics: Mechanical properties of the take-off leg as a support mechanism in the long jump. Sports Biomechanics, 2005; 4: 1-15

Nunn-Cearns G. Long Jump. Modern Athlete \& Coach, 2011; 49: 12-14

Panoutsakopoulos V, Kollias IA. 3D Biomechanical Analysis of Women's High Jump Technique. New Studies in Athletics, 2012; 27: 31-44

Ritzdorf W. Approaches to technique and technical training in the high jump. New Studies in Athletics, 2009; 24: $31-34$ 
Schiffer J. The high jump. New Studies in Athletics, 2009; 34: 9-22

Schinkel-Ivy A, Burkhart TA, Andrews DM. Differences in distal lower extremity tissue masses and mass ratios exist in athletes of sports involving repetitive impacts. J Sports Sci, 2014; 32: 533-41

Shepherd J. Long Jump Technique. Track Coach, 2013; 202: 6437-6440

Van Gheluwe B, Roosen P, Desloovere K. Rear foot kinematics during initial takeoff of elite high jumpers: Estimation of spatial position and orientation of subtalar axis. Journal of Biomechanics, 2003; 19: 13-27

Zhao BJ. Analysis on the characteristics of the age and technique in the world men long jump. Zhejiang Sports Science, 2000; 22: 27-30

\section{Corresponding author:}

\section{Petr Kutáč}

Human Motion Diagnostic Center

Varenska 40a 70200

University of Ostrava

Ostrava, Czech Republic

Telephone Number: +420 606276

Fax Number: +420 595628102

E-mail: petr.kutac@osu.cz 